\title{
THE SIGNATURE OF INITIAL CONDITIONS ON MAGNETOHYDRODYNAMIC TURBULENCE
}

\author{
V. Dallas and A. Alexakis \\ Laboratoire de Physique Statistique, École Normale Supérieure, Université Pierre et Marié Curie, Université Paris Diderot, \\ CNRS, 24 rue Lhomond, F-75005 Paris, France; vdallas@1ps.ens.fr, alexakis@lps.ens.fr \\ Received 2014 April 8; accepted 2014 May 23; published 2014 June 5
}

\begin{abstract}
We demonstrate that the initial correlation between velocity and current density fluctuations can lead to the formation of enormous current sheets in freely evolving magnetohydrodynamic (MHD) turbulence. These coherent structures are observed at the peak of the energy dissipation rate and are the carriers of long-range correlations despite all of the nonlinear interactions during the formation of turbulence. The size of these structures spans our computational domain, dominating the scaling of the energy spectrum, which follows a $E \propto k^{-2}$ power law. As the Reynolds number increases, the curling of the current sheets due to Kelvin-Helmholtz-type instabilities and reconnection modifies the scaling of the energy spectrum from $k^{-2}$ toward $k^{-5 / 3}$. This transition occurs due to the decorrelation of the velocity and the current density which is proportional to $\operatorname{Re}_{\lambda}^{-3 / 2}$. Finite Reynolds number behavior is observed without reaching a finite asymptote for the energy dissipation rate even for a simulation of $\operatorname{Re}_{\lambda} \simeq 440$ with $2048^{3}$ grid points. This behavior demonstrates that even state-of-the-art numerical simulations of the highest Reynolds numbers can be influenced by the choice of initial conditions and consequently they are inadequate to deduce unequivocally the fate of universality in MHD turbulence. Implications for astrophysical observations are discussed.
\end{abstract}

Key words: magnetohydrodynamics (MHD) - turbulence

\section{INTRODUCTION}

Turbulence is a multi-scale phenomenon ubiquitous in numerous astrophysical phenomena (Cho et al. 2003). The magnetohydrodynamic (MHD) description of astrophysical plasmas is often invoked to theoretically model the spectra of velocity and magnetic fluctuations. The precise power law scaling of these fluctuations has implications for the prediction of the heating rates of the solar corona (Cranmer \& van Ballegooijen 2003), the acceleration of the solar wind (Verdini \& Velli 2007; McIntosh et al. 2011), the transport of mass and energy into the Earth's magnetosphere (Sundkvist et al. 2005), the dynamics of the interstellar medium (Spangler \& Cordes 1998; Gaensler et al. 2011), etc.

At high enough Reynolds numbers, a universal power law of the energy spectrum is expected to be observed in strong MHD turbulence. However, to date, theory, simulations, and observations have been unable to provide a definitive answer to the power-law scaling in the inertial range of strong MHD turbulent flows (Iroshnikov 1964; Kraichnan 1965; Goldreich and Sridhar 1995; Müller \& Grappin 2005; Boldyrev 2005; Mininni \& Pouquet 2007; Podesta et al. 2007; Lee et al. 2010; Beresnyak 2012). The power-law scaling exponents $-2,-5 / 3$, and $-3 / 2$ have been observed in various studies of MHD turbulence for both isotropic and anisotropic energy spectra. Thus, universality in MHD has been questioned by many authors (Schekochihin et al. 2008; Lee et al. 2010; Mininni 2011; Wan et al. 2012; Dallas \& Alexakis 2013a, 2013c) in terms of various arguments such as dependence on initial conditions, non-locality, strong anisotropy, and lack of self-preservation.

In this Letter, we present the impact of initial conditions on freely evolving, homogeneous MHD turbulence. We show that coherent flow structures can actually be the carriers of initial cross-correlations and thus imply long-range effects of initial conditions that can influence turbulent statistics such as the power-law scaling of the energy spectrum. Moreover, we demonstrate that correlations between the velocity and the current field are responsible for the $k^{-2}$ energy spectrum in strong MHD turbulence. Our work also indicates that numerical simulations with higher Reynolds numbers than those feasible at the moment are imperative in order to avoid any finite Reynolds number effects and to be able to infer unequivocally if strong MHD turbulence is universal or not. Moreover, our results suggest an alternative scenario for interpreting the $k^{-2}$ energy spectrum observed in the Jovian magnetosphere (Saur et al. 2002).

\section{NUMERICAL SET-UP}

To demonstrate these points, we perform high-resolution simulations of the incompressible MHD equations,

$$
\begin{aligned}
& \left(\partial_{t}-v \boldsymbol{\Delta}\right) \boldsymbol{u}=(\boldsymbol{u} \times \boldsymbol{\omega})+(\boldsymbol{j} \times \boldsymbol{b})-\nabla P, \\
& \left(\partial_{t}-\kappa \boldsymbol{\Delta}\right) \boldsymbol{b}=\nabla \times(\boldsymbol{u} \times \boldsymbol{b}),
\end{aligned}
$$

with $\boldsymbol{u}$ being the velocity, $\boldsymbol{b}$ being the magnetic field, $\omega \equiv \boldsymbol{\nabla} \times$ $\boldsymbol{u}$ being the vorticity, $\boldsymbol{j} \equiv \boldsymbol{\nabla} \times \boldsymbol{b}$ as the current density, $P$ as the pressure, $v$ as the kinematic viscosity, and $\kappa$ as the magnetic diffusivity. If $v=\kappa=0$, then the total energy $E \equiv(1 / 2)\left\langle|\boldsymbol{u}|^{2}+|\boldsymbol{b}|^{2}\right\rangle$, the cross helicity $H_{c} \equiv\langle\boldsymbol{u} \cdot \boldsymbol{b}\rangle$, and the magnetic helicity $H_{b} \equiv\langle\boldsymbol{a} \cdot \boldsymbol{b}\rangle$ are conserved in time, where $\boldsymbol{b} \equiv \boldsymbol{\nabla} \times \boldsymbol{a}$ and $\boldsymbol{a}$ is the solenoidal magnetic potential. Note that the angle brackets $\langle$.$\rangle here denote spatial averages. Using$ the pseudo-spectral method, we numerically solve Equations (1) and (2) in a three-dimensional periodic box of size $2 \pi$, satisfying $\boldsymbol{\nabla} \cdot \boldsymbol{u}=\boldsymbol{\nabla} \cdot \boldsymbol{b}=0$. Aliasing errors are removed using the $2 / 3$ de-aliasing rule, i.e., wavenumbers $k_{\min }=1$ and $k_{\max }=N / 3$, where $N$ is the number of grid points in each Cartesian coordinate. For more details on the numerical code, see Gómez et al. (2005) and Mininni et al. (2011). 
Table 1

Numerical Parameters and Values Obtained at the Peak of the Energy Dissipation Rate

\begin{tabular}{lcrrrrr}
\hline \hline$N$ & $v=\kappa$ & \multicolumn{1}{c}{$\operatorname{Re}$} & $\operatorname{Re}_{\lambda}$ & $u^{\prime}$ & $b^{\prime}$ & $k_{\max } \eta$ \\
\hline 128 & $5 \times 10^{-3}$ & 178.0 & 68.5 & 0.73 & 1.02 & 1.23 \\
256 & $3 \times 10^{-3}$ & 273.0 & 94.3 & 0.73 & 1.04 & 1.74 \\
512 & $1 \times 10^{-3}$ & 639.2 & 166.3 & 0.72 & 0.98 & 1.56 \\
1024 & $4 \times 10^{-4}$ & 1550.3 & 298.6 & 0.75 & 1.02 & 1.62 \\
2048 & $2 \times 10^{-4}$ & 2972.5 & 438.0 & 0.73 & 1.04 & 1.98 \\
\hline
\end{tabular}

The resolutions that we report in this letter range from $N=$ 128 to $N=2048$ (see Table 1 ). The Reynolds number based on the integral length scale $L \equiv(3 \pi / 4) \int k^{-1} E(k) d k / \int E(k) d k$ is $\operatorname{Re}=u^{\prime} L / v$ and that based on the Taylor microscale $\lambda \equiv\left(5 \int E(k) d k / \int k^{2} E(k) d k\right)^{1 / 2}$ is $\operatorname{Re}_{\lambda}=u^{\prime} \lambda / v$, where $u^{\prime}=\left\langle|\boldsymbol{u}|^{2}\right\rangle^{1 / 2}$ is the rms velocity. The smallest length scale in our flows is defined based on Kolmogorov scaling $\eta \equiv\left(v^{3} / \epsilon\right)^{1 / 4}$, where $\epsilon \equiv v\left\langle|\omega|^{2}\right\rangle+\kappa\left\langle|\boldsymbol{j}|^{2}\right\rangle$ is the total energy dissipation rate. The time we address in our analysis is $t_{\text {peak }}$, defined as the time of maximum $\epsilon$, when the highest scale separation occurs $L \gg \ell \gg \eta$ where $\ell$ is a typical length scale in the inertial range. Thus, the values provided in Table 1 correspond to $t_{\text {peak }}$.

Previous work on MHD flows with Taylor-Green (TG) symmetries (Dallas \& Alexakis 2013b, 2013c) has demonstrated that a -2 power-law scaling of the energy spectrum can originate from magnetic discontinuities corresponding to strong amplitude current sheets. We notice that in these flows $\boldsymbol{j}$ initially satisfied the same TG symmetries with $\boldsymbol{u}$ and $\boldsymbol{b}$ with $\boldsymbol{\omega}$. So, here we consider random initial conditions with strong crosscorrelation between the velocity and the current density by setting $\boldsymbol{j} \propto \boldsymbol{u}$ in order to investigate if the $k^{-2}$ spectrum is a more general solution and not only an effect of the TG symmetries (Dallas \& Alexakis 2013c). Initially, our fields are excited at wavenumbers $k=1$ and 2 with random phases and they are normalized such that the kinetic energy $E_{u} \equiv(1 / 2)\left\langle|\boldsymbol{u}|^{2}\right\rangle$ and the magnetic energy $E_{b} \equiv(1 / 2)\left\langle|\boldsymbol{b}|^{2}\right\rangle$ are in equipartition (namely, $\left.E_{u}=E_{b}=0.5\right)$ and all the helicities are zero including the kinetic helicity $H_{u} \equiv\langle\boldsymbol{u} \cdot \boldsymbol{\omega}\rangle$. Note that the magnetic Prandtl number is unity (i.e., $v=\kappa$ ) for all the simulations.

\section{RESULTS AND DISCUSSION}

Here, we compare our results with the results by Mininni \& Pouquet (2009), who carried out direct numerical simulations (DNSs) by superposing Fourier modes with random phases of initially uncorrelated fields for $N=64$ to $N=1536$ (see Mininni \& Pouquet 2009 for more details). In Figure 1(a), we plot the dissipation coefficient $C_{\epsilon} \equiv \epsilon L_{0} / u_{0}^{\prime}$ for each $\operatorname{Re}_{\lambda}$, where the value of $\epsilon$ is taken at $t_{\text {peak }}$ and the integral length scale $L_{0}$ as well as the rms velocity $u_{0}^{\prime}$ are at time zero. The circles represent our runs (see Table 1) and the triangles represent the runs by Mininni \& Pouquet (2009). At high enough $\operatorname{Re}_{\lambda}, C_{\epsilon}$ is expected to asymptote to a finite value. This is the so-called dissipation anomaly of three-dimensional turbulence (Frisch 1995; Biskamp 2003), which is confirmed from the data by Mininni \& Pouquet (2009) with an asymptotic regime from $\operatorname{Re}_{\lambda}>200$ (see Figure 1(b)). In this regime, $C_{\epsilon}=$ const and $\operatorname{Re}_{\lambda} \propto \operatorname{Re}^{1 / 2}$ as expected for a fully developed turbulent flow that obeys Kolmogorov scaling (Frisch 1995). On the other hand, our runs with initial $\boldsymbol{j} \propto \boldsymbol{u}$ cross-correlation have different high Reynolds number asymptotics without reaching an asymptotic regime for $C_{\epsilon}$ even for the highest $\operatorname{Re}_{\lambda}$ run with $2048^{3}$ grid points (note that this numerical simulation is at the cutting-edge
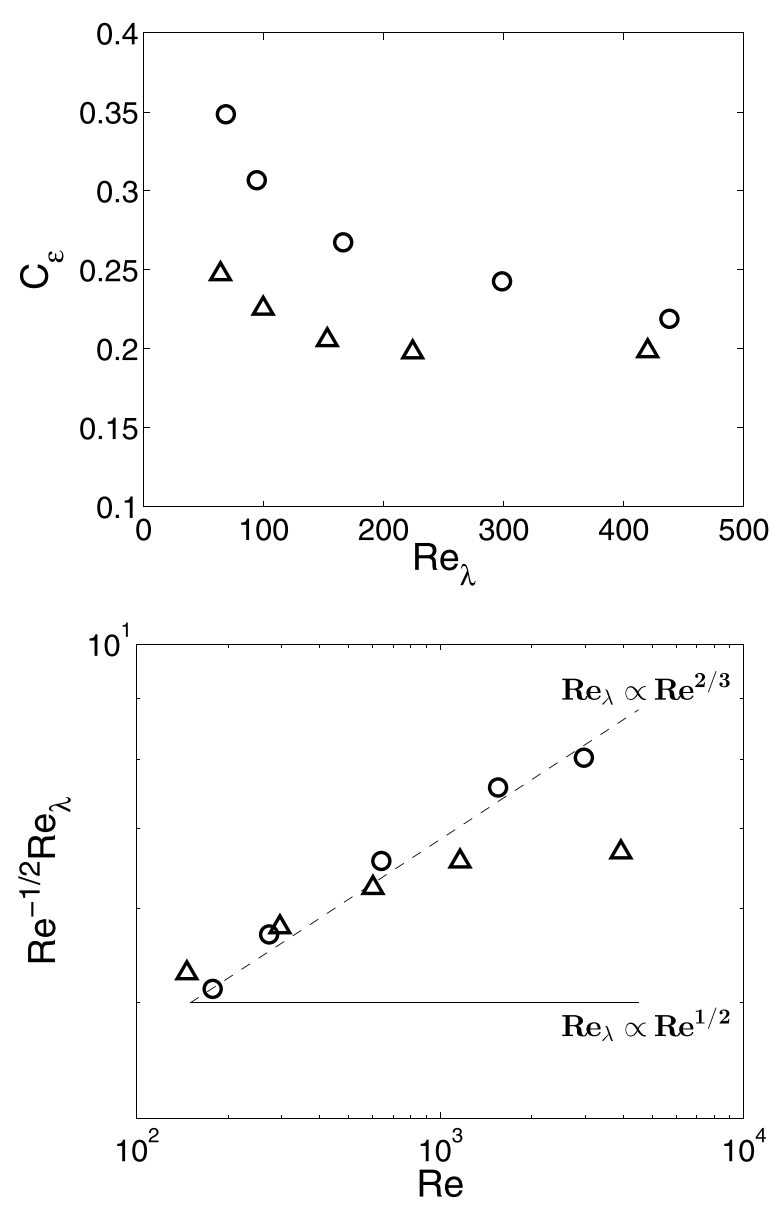

Figure 1. Top: dissipation rate coefficient $C_{\epsilon}$ as a function of $\operatorname{Re}_{\lambda}$. Bottom: $\operatorname{Re}_{\lambda}$ compensated by $\operatorname{Re}^{-1 / 2}$ vs. Re. The circles $\bigcirc$ and the triangles $\triangle$ represent our data (see Table 1) and the data from Mininni \& Pouquet (2009), respectively.

of the current computational capabilities). It is interesting that the $\operatorname{Re}_{\lambda}$ seems to obey a different power-law scaling of the form $\operatorname{Re}_{\lambda} \propto \mathrm{Re}^{2 / 3}$ for this range of parameters. So, a question that arises at this point is if this is a manifestation of non-classical scalings or a finite Reynolds number effect.

High-resolution DNSs of MHD turbulence consist of a large population of intense, dissipative, space-filling sheetlike structures in a wide range of length scales. This large population also includes some structures that remain sparse but become thinner as $C_{\epsilon}$ becomes independent of $v$ and $\kappa$ with increasing Reynolds numbers (see also Mininni \& Pouquet 2009). Visualizations of the current density amplitude in a slice of the entire computational domain are illustrated in Figure 2 at $t_{\text {peak }}$ for the four highest $\operatorname{Re}_{\lambda}$ runs of Table 1 . In contrast to conventional DNS, we observe enormous current sheets that span the size of our periodic boxes. Note that these coherent structures are not space filling with a thickness of order $\eta$ and spanwise length of order $L$. As $\operatorname{Re}_{\lambda}$ increases, curling of these current sheets takes place as a result of Kelvin-Helmoltz-type instabilities until they become unstable and reconnect. Such instabilities have been recently observed in the large population of current sheets of typical random MHD turbulent simulations (Mininni \& Pouquet 2009) but also at the large scales of solar wind observations (Hasegawa et al. 2004). It is remarkable that these sheets are so coherent that the appearance of a large population of smaller space-filling intense dissipative structures is observed only for the highest $\operatorname{Re}_{\lambda}$ simulation after reconnection. 


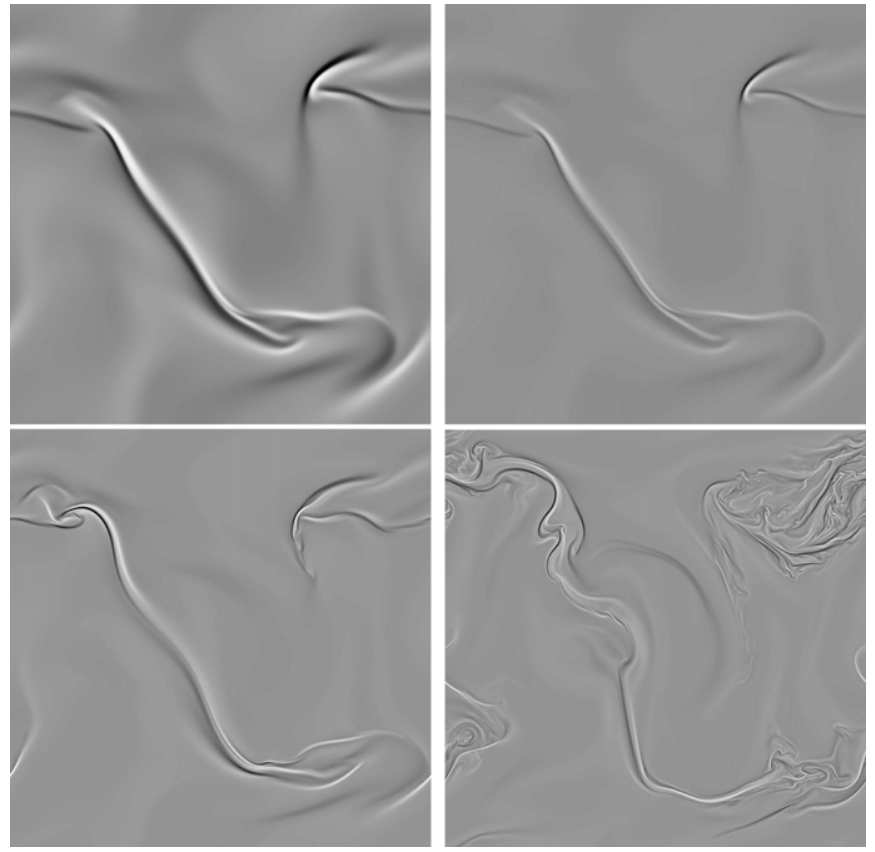

Figure 2. Current density in a slice of the entire box at the peak of the energy dissipation rate for the runs of Table 1. Top left: $\operatorname{Re}_{\lambda}=94.3$, top right: $\operatorname{Re}_{\lambda}=166.3$, bottom left: $\operatorname{Re}_{\lambda}=298.6$, and bottom right: $\operatorname{Re}_{\lambda}=438$. Highand low-intensity regions are denoted by white and black colors, respectively.

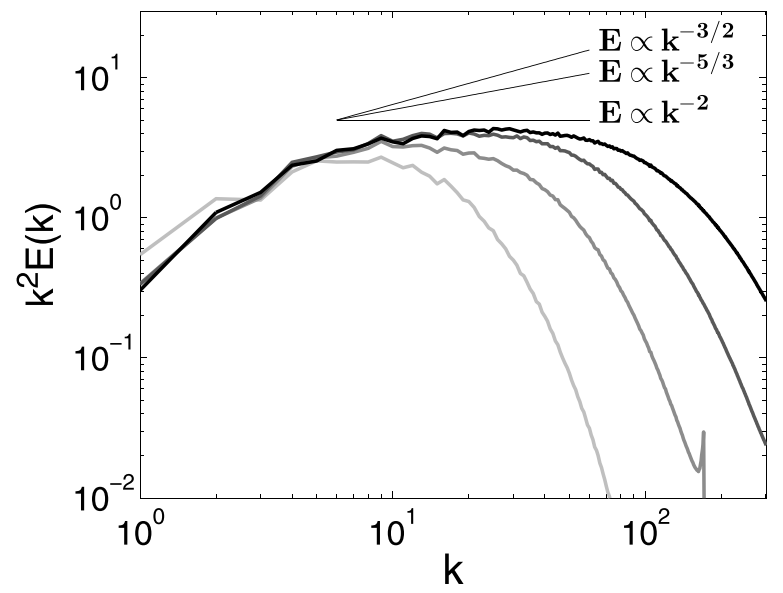

Figure 3. Total energy spectrum $E(k)$ compensated by $k^{2}$ for the four highest $\operatorname{Re}_{\lambda}$ simulations of Table 1 .

Figure 3 presents the compensated total energy spectra $k^{2} E(k)$ of the fields shown in Figure 2 with the power laws $k^{-2}, k^{-5 / 3}$, and $k^{-3 / 2}$ denoted in the plot. The amplitude of these largescale current sheets is strong enough to dominate the energy spectrum, something that is not typically observed in DNS of freely evolving MHD turbulence with random initial conditions. The total energy spectrum seems to form a $k^{-2}$ scaling at least for the runs in which the large-scale current sheets are still coherent. As the Reynolds number increases, the spectrum slowly deviates from the $k^{-2}$ power law toward the $k^{-5 / 3}$ scaling. At high enough Reynolds numbers, we anticipate that either the $k^{-5 / 3}$ or the $k^{-3 / 2}$ will be reached. This result is in agreement with Dallas $\&$ Alexakis (2013c), who demonstrated that the -2 power-law scaling of the energy spectrum will deviate toward the $-5 / 3$ or the $-3 / 2$ scaling exponents when TG symmetries break at Re $\gg 1$. Note, however, that our result here belongs to a more

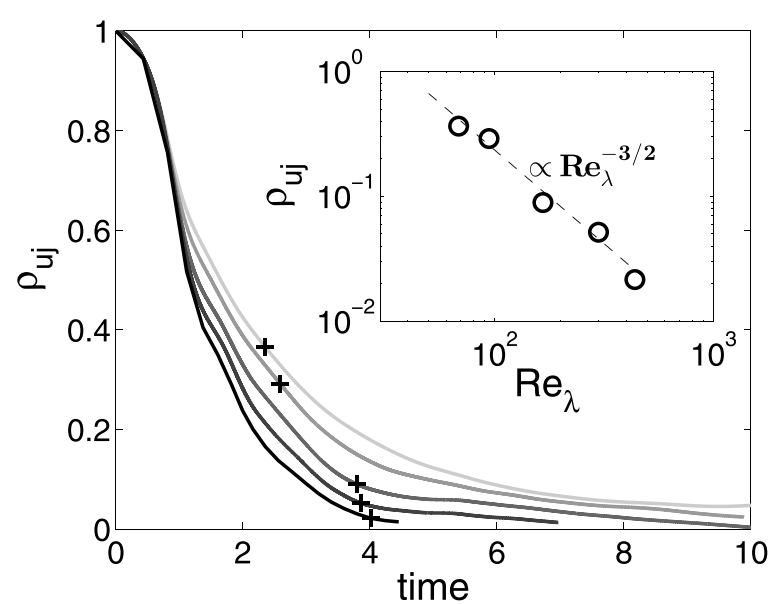

Figure 4. Time evolution of the correlation coefficient $\rho_{u j}$ between the velocity and the current density for all the runs of Table 1 . The light gray curve denotes the lowest $\operatorname{Re}_{\lambda}$ run and the black curve the highest $\operatorname{Re}_{\lambda}$ run. The crosses + indicate the value of $\rho_{u j}$ at the time of maximum dissipation rate $t_{\text {peak }}$. The inset presents the values of $\rho_{u j}$ at $t_{\text {peak }}$ for all the different $\operatorname{Re}_{\lambda}$ runs.

general class of flows with random initial conditions without any symmetry constraints, allowing turbulence to evolve freely.

A measure of quantifying the effect of the initial conditions $\boldsymbol{j} \propto \boldsymbol{u}$ on the flow is the time evolution of the correlation coefficient between the velocity and the current density $\rho_{u j}=\langle\boldsymbol{u} \cdot \boldsymbol{j}\rangle /\left(\left\langle|\boldsymbol{u}|^{2}\right\rangle\left\langle|\boldsymbol{j}|^{2}\right\rangle\right)^{1 / 2}$ that we show in Figure 4. Initially, the velocity and the current density are fully correlated (i.e., $\rho_{u j}=1$ ) and then as time evolves $\rho_{u j}$ decays toward zero. Note, however, that this decay rate is faster as $\operatorname{Re}_{\lambda}$ increases. The crosses in Figure 4 indicate the value of $\rho_{u j}$ at the time of the maximum energy dissipation rate. It is clear that for the lower $\operatorname{Re}_{\lambda}$ runs the cross-correlation between $\boldsymbol{u}$ and $\boldsymbol{j}$ is strong with the current sheets having a spanwise length of the order of the box size $2 \pi$ and a $k^{-2}$ power law for the energy spectra (see Figures 2 and 3). For the highest $\operatorname{Re}_{\lambda}$ runs, where the energy spectrum deviates from the $E \propto k^{-2}$ scaling and the current sheets become unstable, breaking down into a large population of smaller scale structures, we observe that $\rho_{u j}$ is negligible compared to its initial value. Furthermore, the inset of Figure 4 presents the values of $\rho_{u j}$ at $t_{\text {peak }}$ for the different $\operatorname{Re}_{\lambda}$ runs of Table 1. The correlation coefficient exhibits a power law of the form $\rho_{u j} \propto \operatorname{Re}_{\lambda}^{-3 / 2}$, demonstrating the rate at which MHD turbulence loses memory of initial conditions.

\section{CONCLUSIONS}

In summary, we performed high-resolution simulations of freely evolving MHD turbulence with initial cross-correlations of the type $\boldsymbol{j} \propto \boldsymbol{u}$. These initial conditions were chosen in order to investigate if the $k^{-2}$ energy spectrum is not just an effect of the TG symmetries (Lee et al. 2010; Dallas \& Alexakis 2013c) in strong MHD turbulence (Dallas \& Alexakis 2013b) but instead a result of these special correlations. We focus on the time when the peak of energy dissipation rate is reached, which is when the largest scale separation occurs. At this point, we observe current sheets with a spanwise length of the order of the box size $2 \pi$ and thickness of the order of the Kolmogorov scale $\eta$. These structures remain coherent in the flow even for high $\operatorname{Re}_{\lambda}$. These enormous and stable current sheets dominate the energy spectrum particularly for our lower resolution runs with a $E \propto k^{-2}$ power-law scaling as the best fit. As soon as the correlation coefficient $\rho_{u j}$ becomes negligible 
with increasing $\operatorname{Re}_{\lambda}$, Kelvin-Helmoltz-type instabilities kick in, entailing the curling of the enormous current sheets that spontaneously break down to smaller scales only for our run with $N=2048^{3}$, modifying the scaling of the energy spectrum from $k^{-2}$ toward $k^{-5 / 3}$. Therefore, we deduce that the $k^{-2}$ energy spectrum manifests as a finite Reynolds number effect due to correlation between $\boldsymbol{u}$ and $\boldsymbol{j}$. Then, the debate on universality in strong MHD turbulence returns to the distinction between the $k^{-5 / 3}$ and the $k^{-3 / 2}$ scalings at high enough Reynolds numbers.

Overall, our work emphasizes the importance of crosscorrelation between the large scales of $\boldsymbol{u}$ and the small scales of $\boldsymbol{b}$ in freely evolving MHD turbulence. The rate at which the crosscorrelation weakens is relatively slow as $\operatorname{Re}_{\lambda}$ increases (i.e., $\rho_{u j} \propto \operatorname{Re}_{\lambda}^{-3 / 2}$ ) for the resolutions that can be achieved using today's most powerful supercomputers. We anticipate that for higher $\operatorname{Re}_{\lambda}$, the energy dissipation rate will reach an asymptote and the scaling $\operatorname{Re}_{\lambda} \propto \operatorname{Re}^{2 / 3}$ will be modified.

The importance of cross-correlations has been reported in various studies (Pouquet et al. 1986; Osman et al. 2011). So, it is central to investigate if cross-correlations of the type we considered in this study exist and can influence the turbulent statistics in astrophysical observations. For example, the crosscorrelation $\langle\boldsymbol{u} \cdot \boldsymbol{j}\rangle$ and the magnetic discontinuities might be a way to interpret the $k^{-2}$ scaling of the energy spectrum in the Jovian magnetosphere (Saur et al. 2002).

Eventually, it is apparent that there is an urgent need for higher resolutions in numerical simulations of strong MHD turbulence to be able to deduce undoubtedly the fate of universality in the high Reynolds number limit. The fact that MHD turbulence retains certain information from the initial conditions at these Reynolds numbers despite all of the complex nonlinear interactions is an important issue that needs further investigation.

We acknowledge P. D. Mininni and A. Pouquet for their data. V.D. acknowledges the financial support from EU-funded Marie Curie Actions-Intra-European Fellowships (FP7-PEOPLE-2011-IEF, MHDTURB, Project No. 299973). The computations were performed using the HPC resources from GENCI-TGCC-CURIE (Project No. x2013056421) and PRACE-FZJ-JUQUEEN (Project name PRA068).

\section{REFERENCES}

Beresnyak, A. 2012, MNRAS, 422, 3495

Biskamp, D. 2003, Magnetohydrodynamic Turbulence (Cambridge: Cambridge Univ. Press)

Boldyrev, S. 2005, ApJL, 626, L37

Cho, J., Lazarian, A., \& Vishniac, E. 2003, in Turbulence and Magnetic Fields in Astrophysics, ed. E. Falgarone \& T. Passo (Lecture Notes in Physics, Vol. 614; Berlin: Springer), 56

Cranmer, S. R., \& van Ballegooijen, A. A. 2003, ApJ, 594, 573

Dallas, V., \& Alexakis, A. 2013a, PhFl, 25, 105106

Dallas, V., \& Alexakis, A. 2013b, PhRvE, 88, 053014

Dallas, V., \& Alexakis, A. 2013c, PhRvE, 88, 063017

Frisch, U. 1995, Turbulence: The Legacy of A. N. Kolmogorov (Cambridge: Cambridge Univ. Press)

Gaensler, B. M., Haverkorn, M., Burkhart, B., et al. 2011, Natur, 478, 214

Goldreich, P., \& Sridhar, S. 1995, ApJ, 438, 763

Gómez, D. O., Mininni, P. D., \& Dmitruk, P. 2005, AdSpR, 35, 899

Hasegawa, H., Fujimoto, M., Phan, T-D., et al. 2004, Natur, 430, 755

Iroshnikov, P. S. 1964, SvA, 7, 566

Kraichnan, R. H. 1965, PhFl, 8, 1385

Lee, E., Brachet, M. E., Pouquet, A., Mininni, P. D., \& Rosenberg, D. 2010, PhRvE, 81, 016318

McIntosh, S. W., De Pontieu, B., Carlsson, M., et al. 2011, Natur, 475, 477

Mininni, P. D. 2011, AnRFM, 43, 377

Mininni, P. D., \& Pouquet, A. 2007, PhRvL, 99, 254502

Mininni, P. D., \& Pouquet, A. 2009, PhRvE, 80, 025401

Mininni, P. D., Rosenberg, D., Reddy, R., \& Pouquet, A. 2011, ParC, 37, 316

Müller, W. C., \& Grappin, R. 2005, PhRvL, 95, 114502

Osman, K. T., Wan, M., Matthaeus, W. H., Breech, B., \& Oughton, S. 2011, ApJ, 741,75

Podesta, J. J., Roberts, D. A., \& Goldstein, M. L. 2007, ApJ, 664, 543

Pouquet, A., Meneguzzi, M., \& Frisch, U. 1986, PhRvA, 33, 4266

Saur, J., Politano, H., Pouquet, A., \& Matthaeus, W. H. 2002, A\&A, 386,699

Schekochihin, A. A., Cowley, S. C., \& Yousef, T. A. 2008, in Computational Physics and New Perspectives in Turbulence, IUTAM Symp. Comput. Phys. and New Perspectives in Turb., Vol. 4, ed. Y. Kaneda, G. M. L. Gladwell, \& R. Moreau (IUTAM Bookseries; Berlin: Springer), 347

Spangler, S. R., \& Cordes, J. M. 1998, ApJ, 505, 766

Sundkvist, D., Krasnoselskikh, V., Shukla, P. K., et al. 2005, Natur, 436, 825

Verdini, A., \& Velli, M. 2007, ApJ, 662, 669

Wan, M., Oughton, S., Servidio, S., \& Matthaeus, W. H. 2012, JFM, 697, 296 